\title{
The Effects of Incongruent Feedback on Bimanual Task Performance
}

\author{
Joel R. Cooper; Matthew M. Wernke; \& Kyle B. Reed \\ University of South Florida
}

\begin{abstract}
Previous studies analyzing the effects of incongruent visual and haptic feedback have found differences in the perceived stiffness of an object depending on what modality was delayed. These studies required only unilateral performance of the individual and did not measure functional task completion. Our study evaluated the effects of incongruent visual and haptic feedback during a bimanual pick and place task within a virtual environment using two Phantom Omnis. Subjects were asked to place three different colored spheres into matching colored baskets under various testing conditions. The testing conditions included various temporal delays in either the haptic feedback, visual feedback or both feedback modalities simultaneously. The amount of time required to complete the task as well as the number of spheres broken were recorded for each trial. The results show that delays in either or both of the feedback modalities had a negative effect on the subject's ability to complete the task. The most detrimental effects on task performance were observed when both feedback modalities were simultaneously delayed $133 \mathrm{~ms}$ for both the completion time and number of broken spheres.
\end{abstract}

Keywords: Incongruent feedback, haptic, bimanual

\section{INTRODUCTION}

Together, technological advances in the fields of robotics, computer science, and haptics have and will continue to change the way people interact with one another. Currently, one or more users can interact with objects, environments, and even other users inside a virtual environment. For instance, the gaming industry allows many users to interact in a shared environment, often times receiving visual feedback of events from a video screen and haptic feedback of events through the controller, typically a vibration [1]. There are many other applications where haptic and visual feedback could be simultaneously relayed to the user. In telerobotic surgery, a surgeon no longer directly interacts with the patient, but bimanually controls a robotic manipulator from a console while watching the resulting robotic movements and interactions with the patient on a video screen [2]. The advantages to performing surgeries under robotic control include smaller incisions that reduce scarring and pain, more precise control through scaling down of surgeons motions, and elimination of hand tremors [2-4]. However, these systems are still in the early stages and have not been widely accepted into practice yet. This could partially be due to the lack of haptic and force feedback on the master side of the robotic system [5]. The benefits of incorporating haptic feedback into robotic surgery systems are currently being evaluated. One study found that incorporating force feedback into surgical simulators enhanced the users performance during more complicated tasks [6]. Other studies have found incorporating force feedback minimizes

*jrcoope4@mail.usf.edu

†mwernke@mail.usf.edu

‡kylereed@usf.edu

IEEE Haptics Symposium 2012

4-7 March, Vancouver, BC, Canada

978-1-4673-0807-6/12/\$31.00 @2012 IEEE error over graphical force feedback or no force feedback during a palpation task [7].

As these systems develop, the possibilities grow. The trend in gaming is already moving to one that is more immersive, where the player no longer controls the avatar though joystick motions, but through actual body gestures [8]. Similarly, the end goal of surgical robots is to become an extension of the surgeon, so operations can occur intuitively as if the surgeon were directly handling the tissues and organs. However, any latency in the visual or haptic feedback provided to the user could negatively affect the performance. Previous work in this area has shown that incongruent visual and haptic feedback affects the perceived stiffness of an object during a unilateral task [9-12]. These studies found that an object's perceived stiffness decreased as haptic feedback was delayed. One of these studies found that, as a subject explored a surface, they tended to feel harder surfaces when the haptic feedback was delayed and softer surfaces when the haptic feedback was early [11]. This study also found subjects consistently overestimated surface stiffness during palpation if the haptic feedback was delayed. Ferrari et al. studied object softness and concluded a delay threshold for haptic information existed between $66.6 \mathrm{~ms}$ and $133.3 \mathrm{~ms}$ that effects a user's perception. These studies only had the subjects explore the objects using one hand and analyzed the accuracy of a perceived object characteristic. The effects of incongruent feedback on functional task performance have yet to be done as well as the effects on bimanual performance.

The purpose for this paper was to report on the effects of incongruent visual and haptic feedback on one's ability to perform a bimanual task. The time to complete a specified task as well as the number of mistakes made was recorded as performance measures for the functional task performance. The authors thought it was interesting to analyze the effects on overall task performance because this type of analysis could offer an easier comparison to robotic surgeries and how the surgeon's perception of surfaces and interactions can be altered. Various temporal delays were introduced into one or both of the feedback modalities. The information gained in this study will be important for developing algorithms to detect and correct for latency in teleoperated feedback systems.

\section{EXPERIMENT DESIGN}

A virtual environment consisting of three different colored spheres and three matching colored baskets was created using Visual Studio 2008 (Figure 1). Users sat in front of a computer monitor displaying the virtual environment and held a Phantom Omni in each hand using their preferred grip. Users interacted with the spheres using the Omnis to bimanually pick up one of the colored balls and placing it in the matching colored basket. While the Omnis were capable of providing force feedback in three dimensions, the visual feedback was restricted to the two dimensions shown on the computer screen. To compensate, a constant force was applied along the axis of the Omni aligned perpendicular to the computer screen to maintain the motion in one plane. The subjects had unrestricted motion of the Omni's in the remaining two degrees of freedom, which matched the visual representation.

The objective for each task was to correctly place the three spheres into the appropriate basket as quickly as possible under various testing conditions. After a sphere was placed in the match- 
ing colored basket, a lid would appear and keep the sphere from exiting the basket and interfering with other spheres (Figure 1). Users were instructed to start with the sphere closest to the baskets (white sphere) and work outward in order to avoid an artificial rise in the number of broken spheres. The testing conditions included 4 different temporal delays introduced into either the haptic, visual, or both modalities simultaneously, combining into twelve combinations, which were repeated 3 times each, totaling 36 trials per subject. While there were 12 combinations of trials under this set up, only 10 of them are distinct trials since a delay of $0 \mathrm{~ms}$ is the same across the three delay categories. The 36 trials
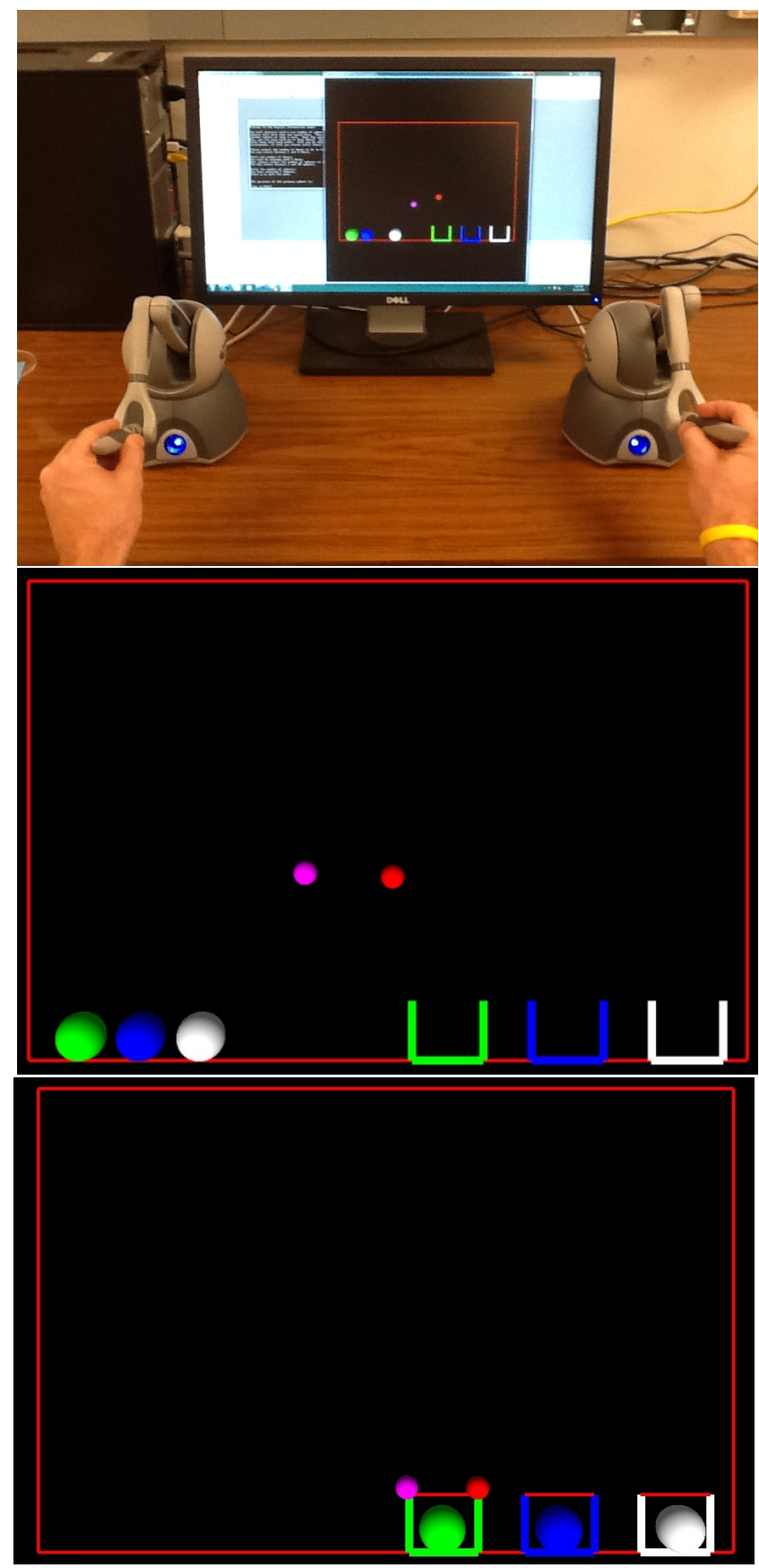

Figure 1: (Top) Point of view of the subject, (Middle) Virtual environment displayed to the subject at beginning of trial, (Bottom) Completed Trial were presented in random order for each subject. Trial conditions were randomized using Excel prior to the subject's arrival and the order recorded for the testing procedures. Based on preliminary data using an earlier version of the program, it was determined that a maximum trial length of three minutes would allow sufficient time for most subjects to complete the trials without affecting the results. The four delay settings were $0 \mathrm{~ms}$ (no delay), $33 \mathrm{~ms}, 67 \mathrm{~ms}$, or $133 \mathrm{~ms}$ delay. Due to the sampling rate of the haptic system, the visual system could only do delays in this amount. The testing procedures typically took about an hour to complete, \pm 0.5 hour.

A number of secondary outcomes were recorded for analysis. First, the number of spheres broken during the task was recorded. The spheres were modeled as fragile objects (e.g., eggs) in order to increase the difficulty of the task. When an interaction force from the user or another sphere exceeded $1.5 \mathrm{~N}$, the sphere would break and reset to its beginning position. For clarification, if two spheres collided, the sphere with the greater absolute velocity would reset to its respective initial position while the other sphere would continue on its previous trajectory. A condition that the same sphere could not break more than once every $200 \mathrm{~ms}$ was established to avoid cases when a broken sphere would respawn in an area with another sphere, causing another broken sphere. Without that condition in place, the broken sphere count would artificially rise. Second, if the maximum trial time of 3 minutes was exceeded, the number of spheres the user had correctly placed in the baskets was recorded. The user was allowed to complete the task using any method they preferred, but most would pick up the spheres from underneath and carry them to the appropriate baskets by balancing it on the haptic interface point (HIP). The HIP is the representation of the Omni's tip in the virtual environment. A multivariable two-way analysis of variance was performed for the ten discrete combinations of type and amount of delay to determine the significance of incongruent feedback on task performance.

Five healthy subjects volunteered to participate in this study. The study was approved by the University of South Florida Institutional Review Board and all subjects signed an informed consent prior to participating in the experiment. The subjects had a wide range of experience using haptic feedback devices. Therefore, before the trials began, each subject was given three practice trials to become familiar with the system. During the practice session, no delay was introduced into the system.

A few main strategies to move the spheres were common among all of the subjects. In one strategy, subjects used both Omnis to pick up the spheres from underneath, move both hands together and drop the sphere into the basket. The second strategy was to use one Omni to pick the sphere up from the bottom and use the other as a spotter to make corrections when needed. A final strategy was to roll the spheres along the bottom and lift the spheres into their respective baskets. The authors recognize 5 subjects is a small sample size; however, even with the small number of subjects, significant results were found. A preliminary study, with slight differences, showed similar results with a slightly higher number of subjects.

\subsection{Force Feedback and Sphere Model}

The Phantom Omnis are impedance devices that input positional data and output a force. When the program is initiated all applied forces on the spheres and Omnis are set to zero. At the end of each $1 \mathrm{~ms}$ cycle, the applied force in the horizontal direction is set to zero while the applied force in the vertical direction is set to simulate gravity. This force was the product of the sphere's mass and the gravitational acceleration constant. Interactions when the HIP came into contact with or penetrated into one of the spheres, the HIP from the other Omni, or one of the baskets, was modeled using the linear spring equation or Hooke's Law, $F=-k x$, where $F$ is the force, $k$ is the spring constant $(0.5 \mathrm{~N} / \mathrm{mm})$, and $x$ is the penetration distance normal to the sphere surface. The unit vector 
describing the position of a sphere or Omni relative to another sphere or Omni is calculated and multiplied by the distance that the objects have penetrated into each other to get $x$. Using Newton's $2^{\text {nd }}$ Law of motion $(F=m a)$, sphere accelerations were calculated using the simulated sphere mass and the combined forces acting on the spheres. The acceleration is then numerically integrated using the trapezoidal integration method to obtain the velocity of the spheres; likewise, the velocity is then numerically integrated to obtain the position of the spheres.

Delays were implemented into the feedback channels by adding an array of zeros to the initial part of the feedback data array. The size of the array added depended on the amount of delay desired. Therefore, the longer the delay needed, the longer the array needed to be. The haptic feedback channel refreshed at a rate of $1000 \mathrm{~Hz}$, but the visual feedback was limited to $60 \mathrm{~Hz}$. Thus, the refresh rate was $1 \mathrm{~ms}$ for the haptic data and $16.7 \mathrm{~ms}$ for the visual data. These values were used as the basis for the time scale to determine the size of the arrays implemented into each feedback type.

A constant coefficient of restitution of 0.9 was chosen for interactions with the spheres, outer limits of the virtual environment, and baskets. A coefficient of restitution that was too large resulted in the spheres bouncing excessively; the opposite effect occurred when the coefficient was too low, thus the authors chose the restitution value that gave the spheres the most natural interactions.

\section{Results}

It is evident from the results that delays in either or both of the feedback signals negatively affected the subjects' performance. The mean completion time and mean number of broken spheres for all subjects based on the trial conditions is shown in Figures 2

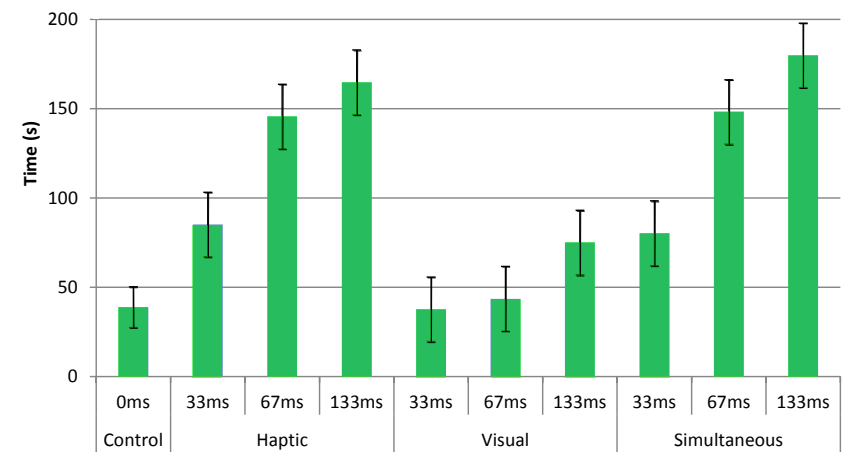

Figure 2: Mean task completion time based on the type and magnitude of delay. The vertical lines represent the $95 \%$ confidence interval.

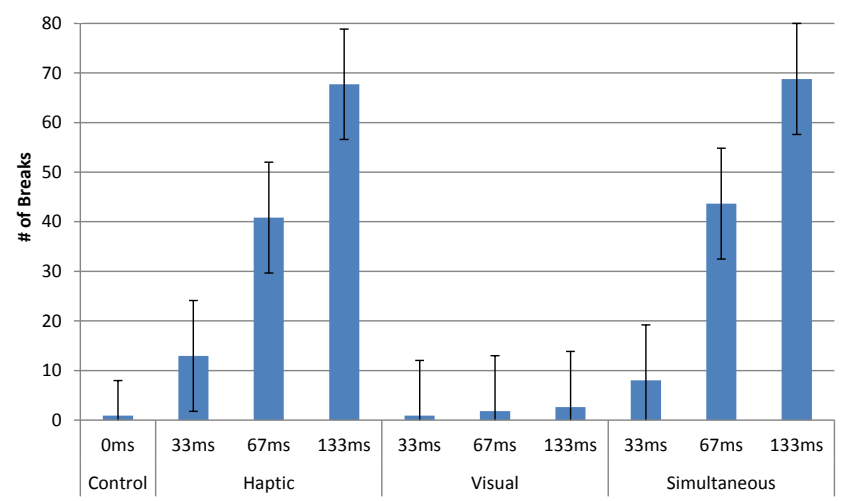

Figure 3: Mean number of broken spheres based on the type and magnitude of delay. The vertical lines represent the $95 \%$ confidence interval. and 3 respectively, along with the standard deviation. The standard deviation (not the confidence interval as shown) for the completion time for the $133 \mathrm{~ms}$ simultaneous haptic and visual delay was zero because the maximum trial time was reached for all subjects and trials. As can be inferred from Figure 2 and found by performing an ANOVA, significant differences were found for seven of the ten discrete combinations of type and magnitude of delay for the task completion time $(F(4,9)=56.08, p<0.001)$. Similarly, Figure 3 suggests and an ANOVA found significant differences for 4 of the 10 discrete combinations for the number of broken spheres $(F(4,9)=35.02, p<0.001)$. Note that the type and magnitude of delay cannot be analyzed as groups in an ANOVA without excluding the control group since the control is inherent within each group and, thus, results in an insufficient rank; intuitively, this can be seen since the control cannot have a non-zero delay nor can it have a delay type associated with it. Therefore, both discrete and individual grouped ANOVAs were completed.

In order to more easily see the effects of type and magnitude of delay, a separate ANOVA was done to analyze just the type and magnitude separately rather than combining the trials into ten discrete combinations. Significant differences were found for the haptic delay as well as the simultaneous haptic and visual delay for the time required to complete the task $(F(4,3)=66.98, p<$ $0.001)$ as shown in Figure 4. Significant differences were also found for all three delay settings for the task completion time $(F(4,3)=40.68, p<0.001)$ as shown in Figure 5. Significant differences were found for haptic and simultaneous delay cases for the number of broken spheres $(F(4,3)=34.71, P<0.001)$ as shown in Figure 6. Only the longest two delay settings (67 and $133 \mathrm{~ms}$ ) had significant differences for the number of broken spheres $(F(4,3)=27.09, p<0.001)$ as shown in Figure 7 .

\section{Discussion}

Introducing delays into either one or both feedback signals simultaneously affected the subject's ability to perform the bimanual pick and place task. Subjects required the longest time to complete the task when either haptic or simultaneous haptic and visual feedback delay was introduced. As expected, the worst performance occurred when both the haptic and visual feedback was simultaneously delayed by $133 \mathrm{~ms}$ for both the completion time and number of spheres broken. This was followed by the $133 \mathrm{~ms}$ haptic feedback delay. Delays in the visual feedback resulted in only small difference in the completion time and number of broken spheres compared to the no delay condition. Three main strategies for moving the spheres were used by the subjects, as outlined in

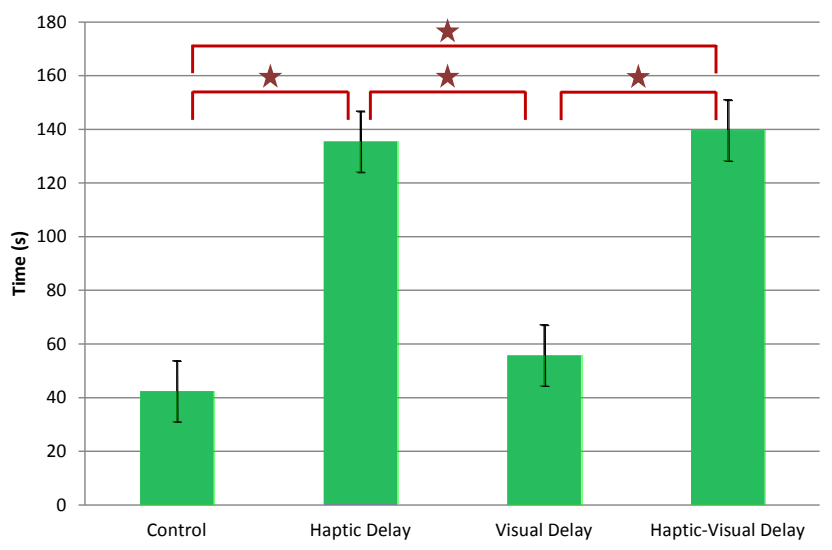

Figure 4: ANOVA results for the type of delay and time to complete the task. The vertical lines represent the $95 \%$ confidence interval and the red lines represent groups that are significantly different. 


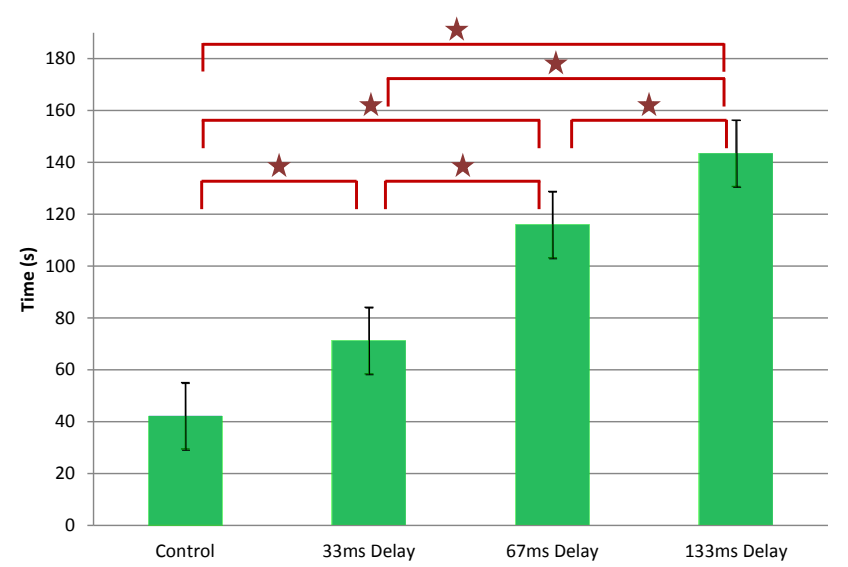

Figure 5: ANOVA results for the magnitude of delay and time to complete the task. The vertical lines represent the 95\% confidence interval and the red lines represent groups that are significantly different.

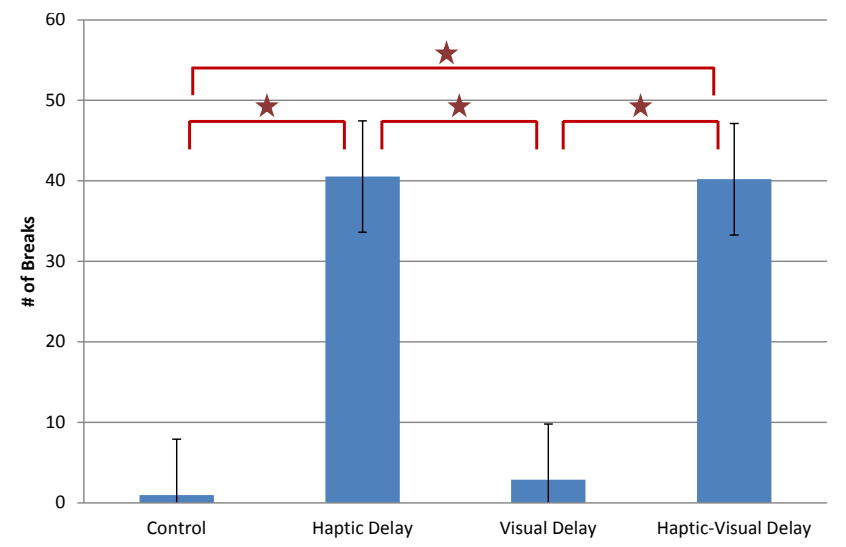

Figure 6: ANOVA results for the type of delay and number of broken spheres. The vertical lines represent the 95\% confidence interval and the red lines represent groups that are significantly different.

Section 2. Typically, subjects primarily used one main strategy while incorporating other strategies on a less frequent basis. Most subjects used the third strategy of rolling the sphere next to the basket before lifting it in. This strategy was probably a favorite among subjects because it reduced the amount of time needed to carry the sphere to the basket.

A maximum trial time of three minutes was set to reduce testing time without affecting the results. In doing so, some of the trial conditions (i.e., the longest two delays for haptic and simultaneous haptic and visual delay) had trials that reached this threshold. When this occurred, the number of spheres that had been placed in the baskets before time expired was recorded. While the results were insignificant, subjects typically were able to place more spheres in the basket under haptic delay than under simultaneous haptic and visual delay. The number of broken spheres was typically the highest for these trials where time expired.

As mentioned earlier, previous studies analyzing the effects on an object's perceived stiffness under active exploration found that a delay in the haptic feedback caused the perceived stiffness of an object to decrease, while the opposite effect was found under visual delay conditions. Even though the emphasis of this study was to determine the effects on overall performance during bimanual operation and not the effects on specific stiffness perception, the results found in this paper seem to correlate with these previous

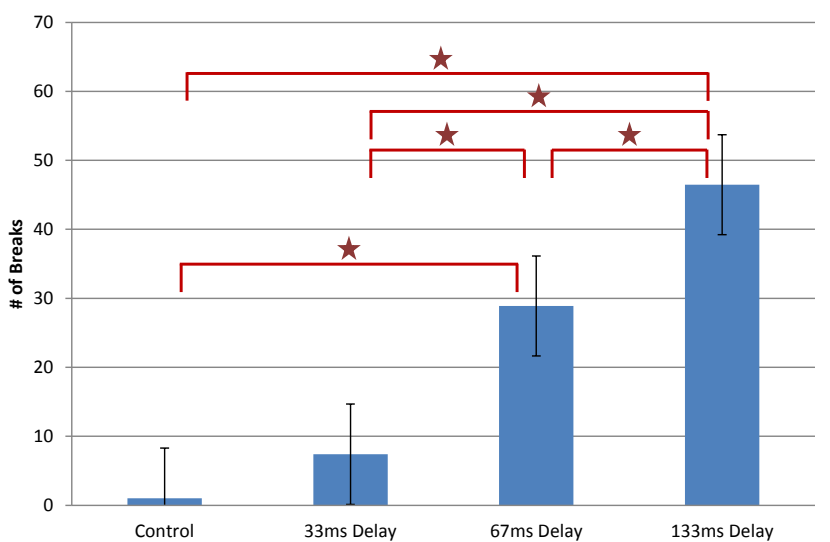

Figure 7: ANOVA results for the magnitude of delay and number of broken spheres. The vertical lines represent the $95 \%$ confidence interval and the red lines represent groups that are significantly different.

studies. A significantly greater number of spheres were broken when haptic feedback was delayed than when visual feedback was delayed. This outcome was due to the fact that subjects were seeing the interaction before feeling it, thus penetrating further into the sphere, increasing the resulting interaction force and exceeding the breaking force threshold of the sphere. The feeling experienced by the subject could be one of interacting with a less stiff object. Alternatively, when visual feedback was delayed, the subjects felt the interaction before seeing it on the screen creating the opposite effect. If a surgeon was performing a teleoperated surgery, delays in haptic feedback could make the surgeon believe his instrument had not contacted the desired tissue since the interaction had not been felt, and potential harm could be done to the tissue or patient and more sutures would be broken. Likewise, in the gaming industry, if a user modeling virtual clay experienced delays in haptic feedback, then the perceived stiffness of the clay would diminish, resulting in changes to the clay the user did not desire.

As you can see from Figure 2, the visual, haptic, and simultaneous delay data approach similar completion times at $133 \mathrm{~ms}, 33 \mathrm{~ms}$, and $33 \mathrm{~ms}$ respectively. Following this, a study could be performed where the delays had smaller steps (e.g., $16 \mathrm{~ms}$ ) to determine the amount of visual delay it would take to reach a similar completion time as the higher level haptic and simultaneous delays, which would effectively find the relative effect of each delay.

While this experimental set up was designed so the subject actively interacted with the spheres, a similar study could be designed where the subject interacted passively with the spheres. This would allow for comparisons to earlier work where the subject passively observed the stiffness of a virtual object and found that delays in the perceived stiffness increased when haptic feedback was delayed.

All five of the subjects were right hand dominant. Even though the task was designed as a bimanual one, all subjects preceded to use their right hand as the primary control for balancing and controlling the sphere. Their left hand was used primarily for keeping the spheres balanced on the right sided HIP. When completing the task under conditions of no delay, subjects were able to "toss the spheres into the baskets. This was done using both Omnis to position the sphere on the right sided HIP and lifting quickly to toss it into the basket. For the hardest trials (133 ms delay in haptic and simultaneous haptic and visual feedback), subjects almost exclusively used their dominant side for controlling the sphere. 


\section{CONClusions ANd Future Work}

The work here suggests further exploration into the effect of incongruent feedback on task performance is needed. First, the expansion of the virtual environment to three dimensions and the addition of frictional properties to the sphere would increase the dependence on haptic feedback since the user could focus on holding the spheres from the side rather than raising the spheres from underneath. Second, further testing with this type of system could lead to the development of an algorithm that predicts a delay and corrects the feedback a user receives. Testing this algorithm would be required in order to determine effectiveness, which would then lead to the development of more complicated systems and their respective algorithms. Including trials with indefinite haptic or visual feedback delay (no force or visual feedback respectively) will help determine the perceived stiffness effects of incongruent feedback. The development of a study that can be completed with no visual feedback could then be used to help develop virtual environments for the visually impaired.

Interacting with objects, environments, and even other users inside a virtual environment is something many fields can benefit from and utilize. In order for these interactions to be as realistic and natural to the user, it is important that any feedback to the user be received correctly. Understanding the potential factors that diminish the fidelity of the virtual interactions will be an important role in developing algorithms to detect potential factors and ultimately correcting them. Further work will help better understand these effects in more complicated system.

\section{REFERENCES}

[1] LaViola, J.J., Bringing VR and Spatial 3D Interaction to the Masses through Video Games. Computer Graphics and Applications, IEEE, 2008. 28(5): p. 10-15.

[2] Mack, M.J., Minimally invasive and robotic surgery. JAMA: The Journal of the American Medical Association, 2001. 285(5): p. 568.

[3] Leddy, L.S., T.S. Lendvay, and R.M. Satava, Robotic surgery: applications and cost effectiveness. Open Access Surgery, 2010. 3: p. 99-107.

[4] Ballantyne, G.H., Robotic surgery, telerobotic surgery, telepresence, and telementoring. Surgical Endoscopy, 2002. 16(10): p. 1389-1402.

[5] Okamura, A.M., Haptic feedback in robot-assisted minimally invasive surgery. Current opinion in urology, 2009. 19(1): p. 102.

[6] Panait, L., et al., The role of haptic feedback in laparoscopic simulation training. Journal of Surgical Research, 2009. 156(2): p. 312-316.

[7] Mahvash, M., Gwilliam, J., Agarwal, R., Vagvolgyi, B., Li-Ming Su, Yuh, D.D., Okamura, A.M.; Force-feedback surgical teleoperator: Controller design and palpation experiments, Symposium on Haptic interfaces for virtual environment and teleoperator systems, 2008.

[8] N. Bianchi-Berthouze, W. Kim, D. Patel, Does body movement engage you more in digital game play and why?, in: A. Paiva, R. Prada, R. W. Picard (Eds.), Affective Computing and Intelligent Interaction, 2007, pp. 102113

[9] Affective Computing and Intelligent Interaction, A. Paiva, R. Prada, and R. Picard, Editors. 2007, Springer Berlin / Heidelberg. p. 102113.

[10] Knörlein, B., Di Luca, M., and Harders, M. (2009). Influence of Visual and Haptic Delays on Stiffness Perception in Augmented Reality. Proceedings of the 8th IEEE International Symposium on Mixed and Augmented Reality, Orlando, FL, 49-52.

[11] Ohnishi, H. and K. Mochizuki, Effect of delay of feedback force on perception of elastic force: a psychophysical approach. IEICE transactions on communications, 2007. 90(1): p. 12-20.

[12] Ferrari, S. and Y. Hu. The effect of incongruent delay on guided haptic training. World Haptics Conference, June 2011. pp. 161 - 166.

[13] Pressman, A., et al., Perception of delayed stiffness. The International Journal of Robotics Research, 2007. 26(11-12): p. 1191. 